\title{
Interactive Documentary on Perspective of New Media
}

\author{
Nayun Kim and Sangheon Kim \\ Dept. of Global Culture and Contents, HUFS, 107 Imun-ro, Seoul, Korea \\ luce728@naver.com,shkim@gcrc.kr
}

\begin{abstract}
An Interactive Documentary is web and multimedia documentary with interactivity and user participatory that present non-linear narratives. These features come from that of new media including hypertext, remediation, modulation and interactivity which is combination of cinema and digital technologies. Focused features of new media and interactive documentary are presented and compared. Variant modes of interactive documentary compared to traditional documentary or media are presented. Interactive Documentary is a New media, mostly affeced by user velated interactions.
\end{abstract}

Keywords: Interactive documentary, new media, remediation, wer interaction

\section{Introduction}

As for film literature, most of them stan with areference to the Lumiere brothers and their cinematographies. AS for interactive docamentary, we should begin with Lumiere's work as well as Babbage's Analytical engine in 1830, the beginning of computers. An interactive documentary thatlies between cinema and interaction is a documentary production that differs from the more traditional forms-video, audio, photographic - by applying a full complement of multimedia tools. The interactive multimedia capability of the Internet provides documentarians with a unique medium to create non-linear productions that combine photography, text, audio, video, animation and info graphics

The time of interactive documentary has come, online video distribution has proven its feasibility on a high bandwidth world and its proximity to interactive environments is pushing it towards interaction. The massive convergence process in course on a battle for "the screen" between TV broadcasters, online newspapers, and media distribution companies that desperately need innovative quality products to survive. Nowadays, everyone is using the word "documentary" to describe every single multimedia piece that incorporates video no matter its nature, technique, language or scope, taking advantage of the fuzzy and fragile boundaries of the documentary definition.

The fundamental difference between a linear and an interactive documentary is not the passage from analogue to digital technology but the passage from linear to interactive narrative. Both linear and interactive documentaries try to create a dialogue with reality, but the media they use afford the creation of different products. A linear documentary that is distributed through the Internet is digital, but if it is not interactive it does not affords new types of construction of reality. The introduction of interactivity, through new media, brings with it new dynamics which, with time, creates new possible aims and therefore new epistemologies.

This paper is composed as follows: We look into principles of New media in Chapter 2 , and summarize features of interactive documentary in Chapter 3, then categorize 
modes of interactivity in interactive documentary in Chapter 4, and make conclusion in chapter 5.

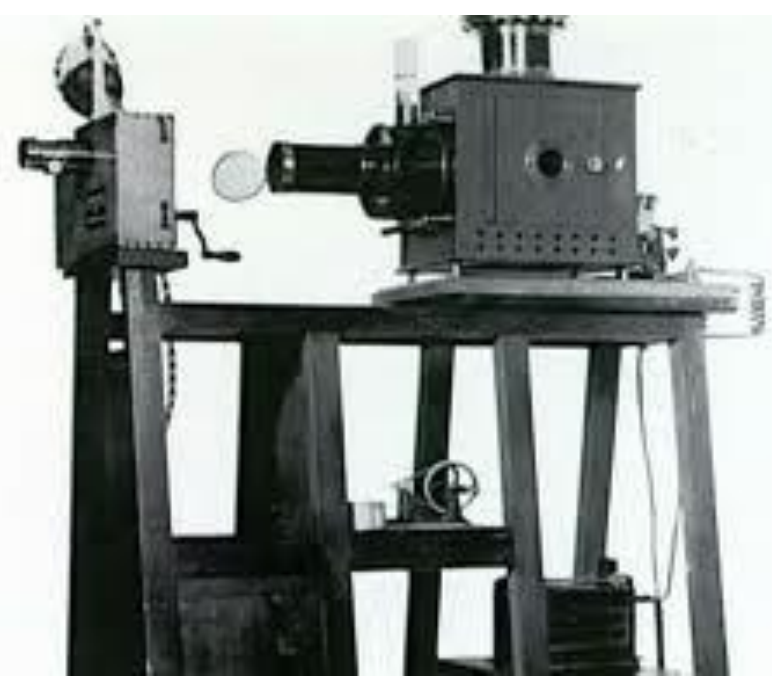

Figure 1. Lumiere's Cinematographie

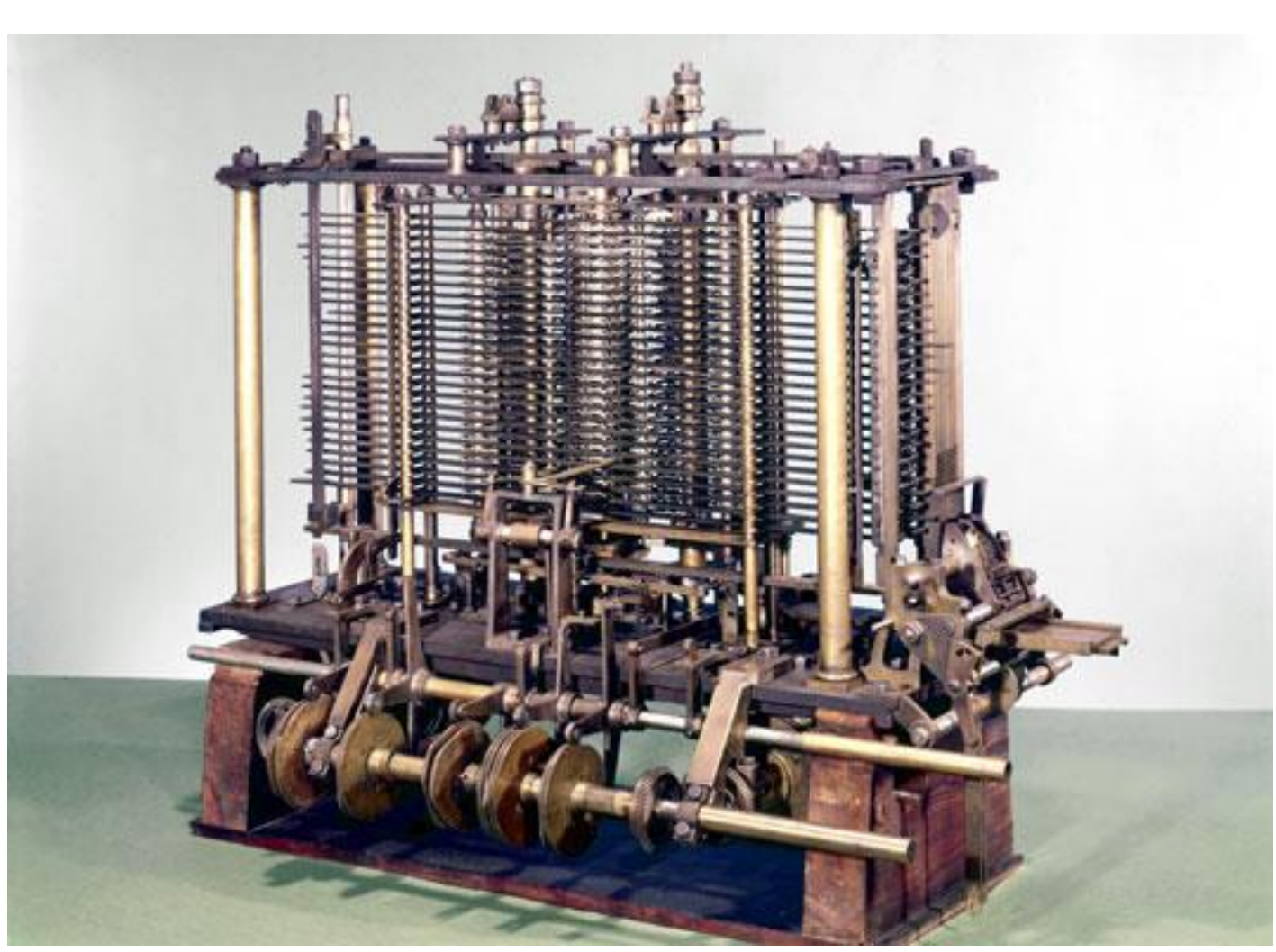

Figure 2. Charles Babbage's Analytical Engine 


\section{Principles of New Media}

According to the Manovich's theories, principles of new media can be considered on followings. 1) Computers as a meta-medium, as a support tool for production and also for consumption of mew media objects. 2) The impact on previous cultural expressions, such as analog cinema or photography, of computing techniques and tools. 3) The influence of software programming paradigms on aesthetics, but even on how we think, learns and access to information. 4) The emergence of a new visual and media culture in our society.

Manovich presented 5 principles of new media summarize as follows.

(1) Numerical representation - For being a new media the object must be represented as a numeric/mathematical expression. - It must be modifiable by an algorithm (a software code to apply some transformations). For example, this is an analog photograph sampled by scanners in what we call a digitization proces. This process turns a continuous surface into sets of discrete unit (the photo and its points) but, like letters in a word, these units construct the object but the are not the meaning itself. Some other mathematical patterns appear in the production side of new media objects. As a modern factory, the process can easily be split in small activitues where different tools/people help to build the object. Here we find a clear difference between modern industrial processes and artisan procedures and we can also extrapolate this from digital to analog.

(2) Modularity - New media adopts fractal stpuctures (I don't agree with this sentence). I rather prefer to compare (t) yyth the structured programming model or the OOP paradigm (Object Oriented Programming) - New media is made of a bunch of components or modules with an ndividual identity. - These components can be mixed in many ways to compose larger objects. They can interact to add some behavior and intelligence and offer them to the end rser, or just in order to be exposed in the same interface. - In a way, modularity principle also talks about composite applications at user layer and distributed systems at systems layer. Examples of this could be mash-ups, they offer a public API to integrate with other services in single user interface and usually in web sites. An API (Application Programming interface) is a communication mechanism (usually employs Web Services approaches) to expose services and data from a Service. The use of this kind of modularity is extended all around de word with services like Google Maps, Facebook, MySpace, GIS Services and more, who give users the possibility to add to their site (embed) some external functionalities. This can be called a top level integration of modular features at the modern Web. Also nonpublic services like enterprise systems use such mechanisms, and most of the new business sofware systems are strongly focused on modularity in all the levels of their subsystems. One of the main SOA principles (Service Oriented Architecture) is loose coupling referred to services independency.

(3) Automation - Very dependent of principle 1 and 2. - Because of the nature of discrete numeric data and modularity of services/modules, new media can be transformed automatically by other processes (usually software programs). - This tends to be done as some kind of AI (Artificial intelligence) in order to generate or manipulate content, or to apply mathematical transformation procedures Example of this could be a computing imaging analysis. Starting from a digitized photograph, a program runs algorithms to find patterns and similarities in a group of photographs, and automate the tedious task of looking for common characteristics. Other interesting examples are Search Engines' Robots. They scan the web looking for web pages in 
order to analyze their contents (words, structure and links) and create a Search Engine Index with relevant information. These indexes allow users to quickly search any topic. Due to the size of the web, this process is impossible to be done outside an automated environment and also impossible with old media. Search indexes are like a transformed version of the original media.

(4) Variability - If a new media could undergo automation processes to generate new versions, obviously the media exists in infinite potential versions. - Variability can be provided also by other drivers, such like user interaction, which represents a one to one communication. The user helps the media to adapt to his/her needs. Examples of this are recommendation systems in ecommerce platforms. What you see is strongly dependent on user behavior at the ecommerce web sites, but not only on a single user. What others buy and what you have bought in previous visits helps de system to offer more specific information, trying to fit with precision your needs and improving conversion rates (visits to sells).

(5) Transcoding - The transformation from one format to another. The change of format implies that the new object can start new conversations with other objects. - This principle talks about the transformation of computing concepts to "cultural" concepts. It generates two different layers: the "cultural layer" and the "computer layer". - Is important to notice the impact of computer layer over cultural layer. - Technology cycles are affecting to the creation and use of "cultural layer objects and vice-versa. And example of this transformation is how old web conmanities (forums, chat, etc.,) evolve into what now we call "social sites", with a high social impact in daily lives. This example portrays how informatics evolution (API, RSS, Mashup services) promotes new "cultural" entities and Innovative üsages.

Manovichi considers the relations between cinema and new media in terms of two vectors. The first one goes from cinema to new media and the second one goes opposite direction from computers to cinema. He summarizes the effects of computerization on cinema as following lists $[1]$.

1 Use of computer techniques in traditional filmmaking

1.1 3-D computer animation/digital composing. Ex) Titanic (James Cameron, 1997, The City of Los Children (Marc Caro and J. P. Jeunet, 1995)

1.2 Digital painting. Ex) Forrest Gump (Robert Zemeckis, 1994)

1.3 Virtual sets. Ex) Ada (Lynn Hershman, 1997)

1.4 Virtual actors/motion capture. Ex) The Lord of the Rings (Peter Jackson, 2001)

2 New forms or computer-based cinema

2.1 Motion rides/location-based entertainment. Ex) rides produced by Douglas Trumbull

2.2 Motion Graphics, or typographic cinema : film + graphic design + typography. Ex) film title sequences.

2.3 Net.cinema : film designed exclusively for internet distribution. Ex) New Venue, one of the first onlines sites devoted to showcasing short digital films.

2.4 Hypermedia interfaces to a film that allows nonlinear access at different scales. Ex) WaxWeb (David Blair, 1994-1999), Stephen Mamber's database interface to Hitchcock's Psycho (Mamber, 1996-)

2.5 Interactive movies and games structured around film-like sequences. Ex) Johnny Mnemonic game, the Blade Runner game) 
2.6 Animated, filmed, simulated, or hybrid sequences that follow film language, and appear in HCI, Web sites, computer games, and other areas of new media. Ex) QuickTime movies in Myst, FMV openings in Tomb Raider.

3 Filmmakers' reactions to the increasing reliance of cinema on computer techniques in postproductions.

3.1 Films by Dogme 95 movement. Ex) Celebration (Vinterberg, 1998)

3.2 Films that focus on the new possibilities offered by DV (Digiral Video) cameras. Ex) Time Code (Figgis, 2000)

4 Filmmakers' reaction to the conventions of new media.

4.1 Conventions of a computer screen. Ex) Prospero's Books (Greenaway)

Conventions of game narratives. Ex) Run, Lola, Run (Tykwer, 1999), Sliding Dooss (Howitt, 1998)

\section{Features of Interactive Documentary}

The Primary material of linear documentary if moving images and so is for interactive documentary. Documentaries are made of images incorporation of other media. Interactive documentary makers have been using pictures as well as moving images. And, regardless the screen, interactive documentary should always pursuit a full screen approach for the sake of immersion and engagement. As far as audio is concerned, it's a key element of interactive documentary due to its versatility and engaging properties.

We can guess some kind of subject is suitable for interactive documentary. Interactivity is not a universal formula 1 oot every subject is suitable for an interactive documentary, on the contrary: we can guess that very few are so every piece needs to start with a firm belief of the subject's suitability to the format. Interactivity is not a universal formula. It's easy to go for the temptation to immediately create an "interactive CD-ROM", or to make a feature length "digital film", instead of focus on determining the new media equivalent of a shot, a sentence, a word, or even a letter". Linear documentary is a successful centennial formula so the question "why not linear" should always come finst, any attemp to force a subject into interactivity is a recipe for failure. Regarding this topic, the approach to the subject must be willing to change as interactivity demands, the elation between format and subject needs to be resilient.

The framework of the interactive documentary should look for formal arrangements within the subject - like geography, chronology, or preferably more sophisticated onesand, more important should run away from the traditional approaches we can find on traditional multinedia products that usually moves the production away from cinema. Formal arrangements shouldn't compel interactive documentary into structural rigidity, it's important to find a solid but flexible structure that allows some degree of freedom. There's also no need to make it too clickable, too interactive: if linear documentary has zerg interaction points and is a successful model, why would we make an interactive one a "clickable extravaganza"? Seek for continuity first, interaction last. And, of course, its always better a good idea made simple.

As far as input devices are concerned, two ideas stand: simplicity and universality. Input devices should be those people know how to operate and they should never surpass the consumer market. The mouse is central to the operation; tactile technology is becoming a standard, too. The microphone is also a good tool to trigger events. Wii is planning on launching a pulse detector so interactive documentaries might incorporate basic biometric data into their input feed. The input can also come from multiple data sources over the Internet, like weather or news reports just to name a few. But, again, 
we emphasize the orientation towards simplicity: every single input should have a grounded purpose and shouldn't be used just because it's fun for the maker. The outcome of the input should be clear to the interaction and significant to the experience, otherwise it should be avoided.

The interface should be moving images based and has to merge with content to the point where one cannot be dissociated from the other. Interface is content, content is interface. Manovich [1] wrote, "contrary to traditional media, in hypermedia the elements and the structure are separate from each other. The structure of hyperlinks typically a branching tree- can be specified independently from the contents of a document". And that's precisely what we should avoid. Smooth continuity between both is compulsory, engagement is a shared responsibility; interactors should "watch" the interface too. Incoherent transitions within and between different narrative threads as one of split-attention stumbling blocks of interactive cinema. So, every clfok should match the previous one. The Tate Street Art website (www.tatestreetart.com) is a great example on how moving images can have engaging properties even, when there's no particular action. Interface should keep away from text and buttons as much as possible (audio cues is a good alternative).

When considering the navigation structure of an interactive documentary it's essential to move away from a linear mindset, any attempt to emulate a linear structure will most certainly fail. An interactive structure has no ability to compete with a rigorous and linear edit made with story arch and closure in mind. Moreover, why giving someone the option to choose if, in a way, it's a blind choice? Can't linear documentary be regarded as a navigation curatorship? And if the goal of some documentaries is to challenge the interactor andits beliefs, should he be able to choose? Some of these issues could be orercome with mput data that wouldn't depend on an explicit choice of the interactor, like biometic data, but it's too early for that.

Consequently, interactive dooumentary should always explore unique approaches to the subject that cinema can achieve the so-called "being there" feeling comes to mind. In that sense, the interactive documentary "Only Fish Shall Visit" is a good model: before the flood of Halfeti due to the construction of a dam, Bunt has decided to document the place under an interactive "flâneur" approach, whereby the interactor wanders through the soon to be underwater town. This technique allows a particular relation with the subject as it comes close to the actual experience of exploring a place, the decisions the interactor make are more or less similar to those he would do if visiting the real town perpetrating a sense of freedom, like choosing the left trail on a junction. This real life emulation generates a strong relation with geography; the choice given to the interactor endures an engaging and memory friendly experience, very useful for pedagogic content. So, the plastic nature of this approach allows exploring some aspects that cinematic montage has been traditionally ignoring for a long time. In a way this approach reminds us a game structure, so it's crucial for interactive documentary to assess what can be merged with other areas and how to perform the operation.

How long should the experience take? Duration is a tricky issue and the answer depends entirely on goal of the work, on how it is supposed to be experienced by the interactor. Some topics should be stressed out, though. First, the replication of the golden rule for video on the Web "keep it short" might not be valid to interactive documentary: if, as we predict, interactive documentary might be consumed on the biggest screen on the living room (note: during leisure time), why can't it be experienced for at least the duration of a linear one? But maybe our mind is too deeply 
connected with linear when it comes to duration, maybe it's an experience that can last for several days if we consider, for instance, that people play video games sometimes for months or even years. Are interactive documentaries supposed to be consumed one time straight or throughout several days? Is the interactor expecting the Aristotelian drama path as usual? Is the closure within the piece or does the interactor needs to find its own closure? Does interactive documentary requires full attention through- out the whole piece? There are too many questions to be answered.

Since interactive documentary is such a new production area and since every single documentary has its own approach then it's normal that the market has no ready-made authoring tools, and we wonder if it will ever have. As a result, interactive documentary has to be built either using general-purpose programming languages, that offer basic or no support for moving image narrativity. Advanced users of the software, of course, trick it, or bend it at least, into doing what they want it to do: but novice users repeat the same structure again and again. If we look closer into the market, probably the best software to author interactive documentaries comes from the video art arena. but these tools are impractical for wide distribution since they don't work as a standalone. Max, for instance, starts with a blank page to which we add-modules, and everything can be connected to everything, because everything speaks numbers. Hence, makers aren't conceptually propelled to follow a specific paradigm allowing a welcoming intellectual freedom. It's important to think ahead, to explore concept before choosing the tool or it will conform thought.

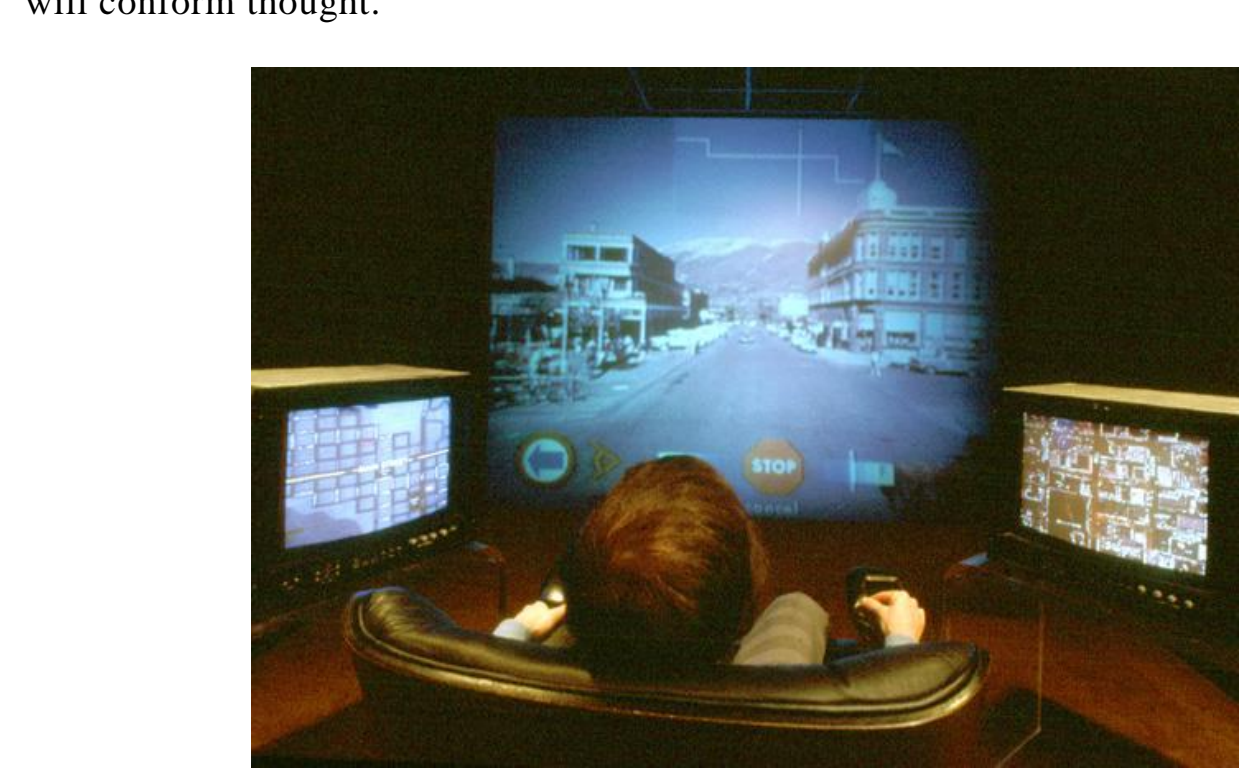

Figure 3. The Aspen Movie Map

\section{Mrodes of Interactivity}

\subsection{The Conversational Mode}

The Aspen Movie Map (Lippman, 1978) is often referred to as the first attempt to digitally document an experience. By using videodisc technology, and three screens, the user was able to drive through a video reconstruction of the city of Aspen. The use of digital technology to simulate a world where the user has the illusion of navigating freely has also been used in video games, MUDs and sandbox games, so it is with no 
surprise that journalists, and new media artists, have been inspired to create 'factual games', or 'docugames', such as Gone Gitmo (Peña, 2007) or Americas Army (Wardynski, 2002). This type of i-doc, which uses 3D worlds to create an apparently seamless interaction with the user, lends itself to the Conversational mode because it positions the user as if 'in conversation' with the computer.

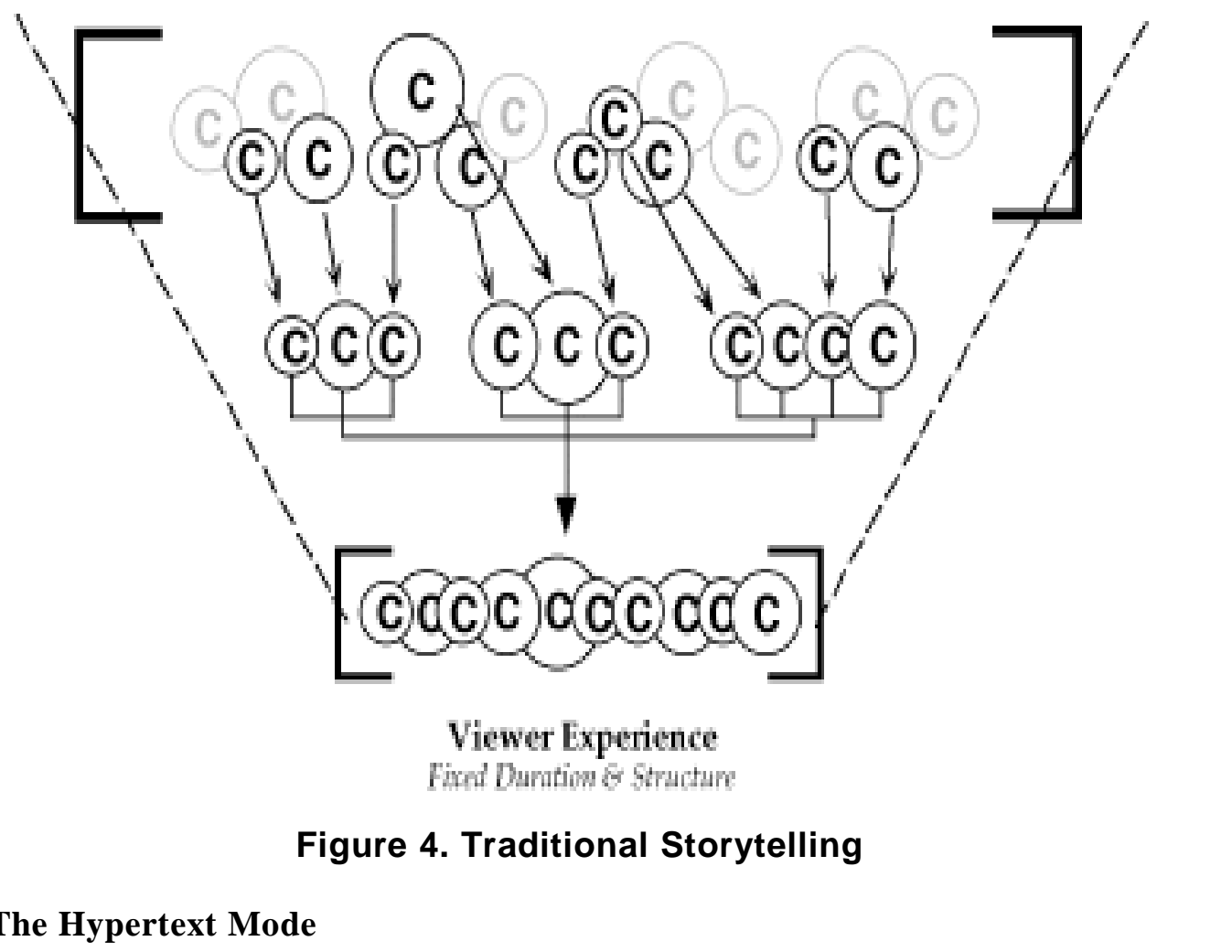

One of the first digital artifacts to be officially called an interactive documentary was Moss Landing (Apple Multimedia Lab, 1989). During one day several cameras recorded the life of the inhabitants of Moss Landing's Harbour. Those assets where then organized as a closed database of video clips that the user could browse via a video hyperlink interface. This logic of hypertext documen- tary has later been applied to CDROMs (such as Inmemory by Marker, 1997) and DVDs (such as Bleeding Through the Layers of Los Angeles by Klein, 2003). Currently a multitude of projects that follow the same logic of 'click here and go there' are being produced for the Web; those are often referred toas, web-docs. Inside The Haiti Earthquake (Gibson and McKenna, 2011), Out My Window (Cizek, 2010), Journey to the End of Coal (Bollendorff, 2009) and Forgotten Flags (Thalhofer, 2007) are just a few examples of this style of inter-active documentary. This type of i-doc lends itself to the Hypertext mode because it links assets within a closed video archive and gives the user an exploratory role, normally enacted by clicking on pre-existing options. 


\subsection{The Participative Mode}

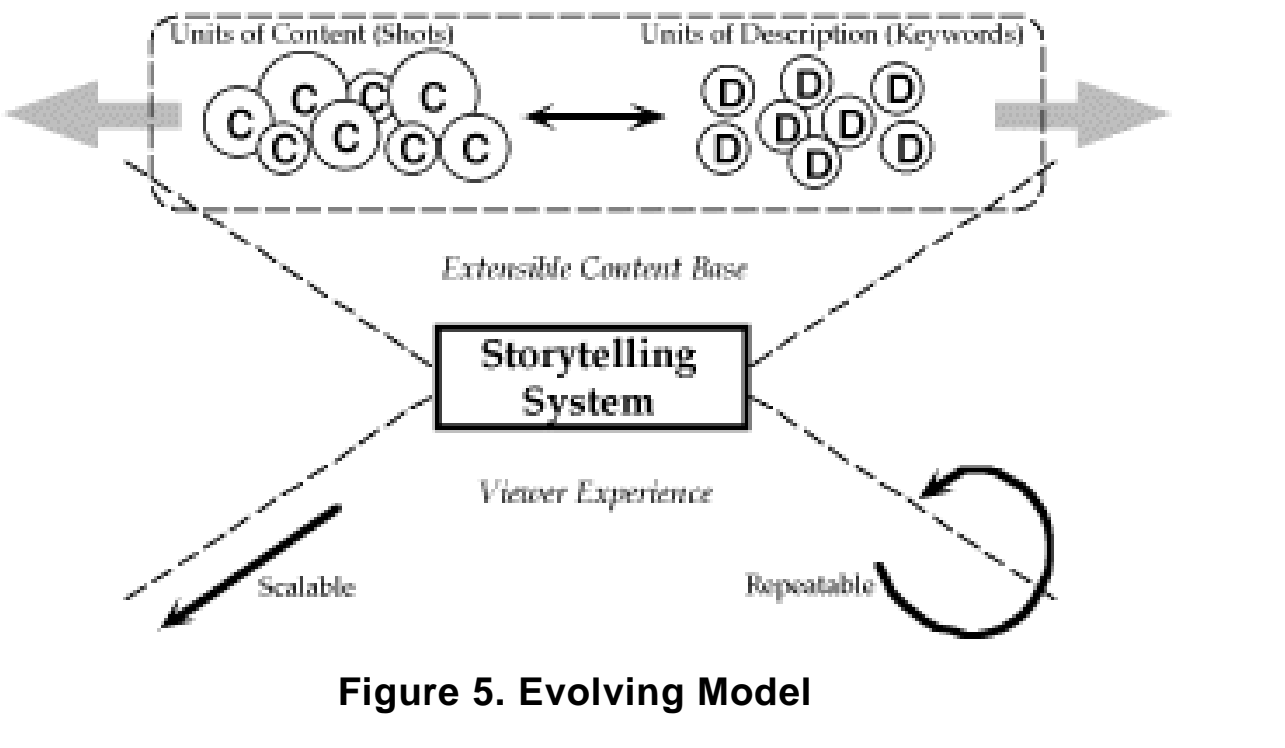

The advent of Web 2.0 has, however, allowed people to go further than browsing through content: the affordances of the media have made possible a two-way relationship between digital authors and their users. Although in the late 1990s the MIT Interactive Cinema Group, led by CHoriana Davenport, tried to develop 'Evolving documentaries' where 'materials grow as the story evolves' (Davenport and Murtaugh, 1995: 6), it was only after 2005. when the penetration of broadband in western countries reached a critical mass, that interactive documentary producers started exploring ways to actively involve thelr users within the production of their digital artefact. In what is often referred to as collab-docs, or participatory-docs, the documentary producer 'is called upon to 'stage a conversation', with a user community, with research subjects, with participants, co-producers and audiences' (Dovey and Rose, forthcoming 2013). In other words, in participative documentaries the user can be involved during the production process - by for example editing online (see RiP: $a$ Remix Manifesto, Gaylor, 2004-2009) or shooting in the streets (see 18 Days in Egypt, Mehta and Elayat, 201) - or during the launch and distri- bution process (e.g. by answering questions online, like in 6 Billion Others (Arthus-Bertrand, 2009), or by sending material and helping translating it as in the Global Lives Project (Harris, 2010). This type of i-doc is described here as being Participative, as it counts on the participation of the user to create an open and evolving database.

\subsection{The Experiential Mode}

Finally, mobile media and The Global Positioning System (GPS) have brought digital content into physical space. 34 North 118 West (Hight, Knowlton and Spellman, 2001), allowed people to walk in the streets of Los Angeles armed with a Tablet PC, a GPS card and headphones. Depending on the position of the participant, stories uncovering the early industrial era of Los Angeles were whispered into the ears of the urban flâneur, accompanied by historic illustrations on the computer screen. In 2007 Blast Theory created Rider Spoke (Adams, 2007), a bicycle ride where people could record very personal answers via the use of a mobile device (Nokia N800) mounted on the 
handlebar of their bicycle. Those testimonies were then made accessible to any other participant passing in the area where the message was first recorded. This type of locative documentary invites the participant to experience a 'hybrid space' (De Souzae Silva 2006: 262) where the distinction between the virtual and the physical becomes blurred. I-docs of this nature tend to play on our enacted perception while moving in space. As the participant moves through an interface that is physical (although enhanced by the digital device) embodiment and situated knowledge are constantly elaborating new situated meanings. This category is named as being Experiential because it brings users into physical space, and creates an experience that challenges their senses and their enacted perception of the world. Definitions of linear documentary have changed over time. The term still means different things to different people. We have followed Bill Nichol's approach [4], using a systemic definition that sees documentary as a set of relations forged between the author, the viewer, the media and what is around them. Those relations are changing with time; they are influenced by social, political and technological change. Those modes are ways to frame and organize (reality) into a text' and therefore they are symptomatic of a modes of 'negotiation' with reality. It is the idea of logics of negotiation of reality that I have retained to analyze digital interactive documentaries claiming that, once the user is demanded an active participation in the documentary, the negotiation happens through interactivity.

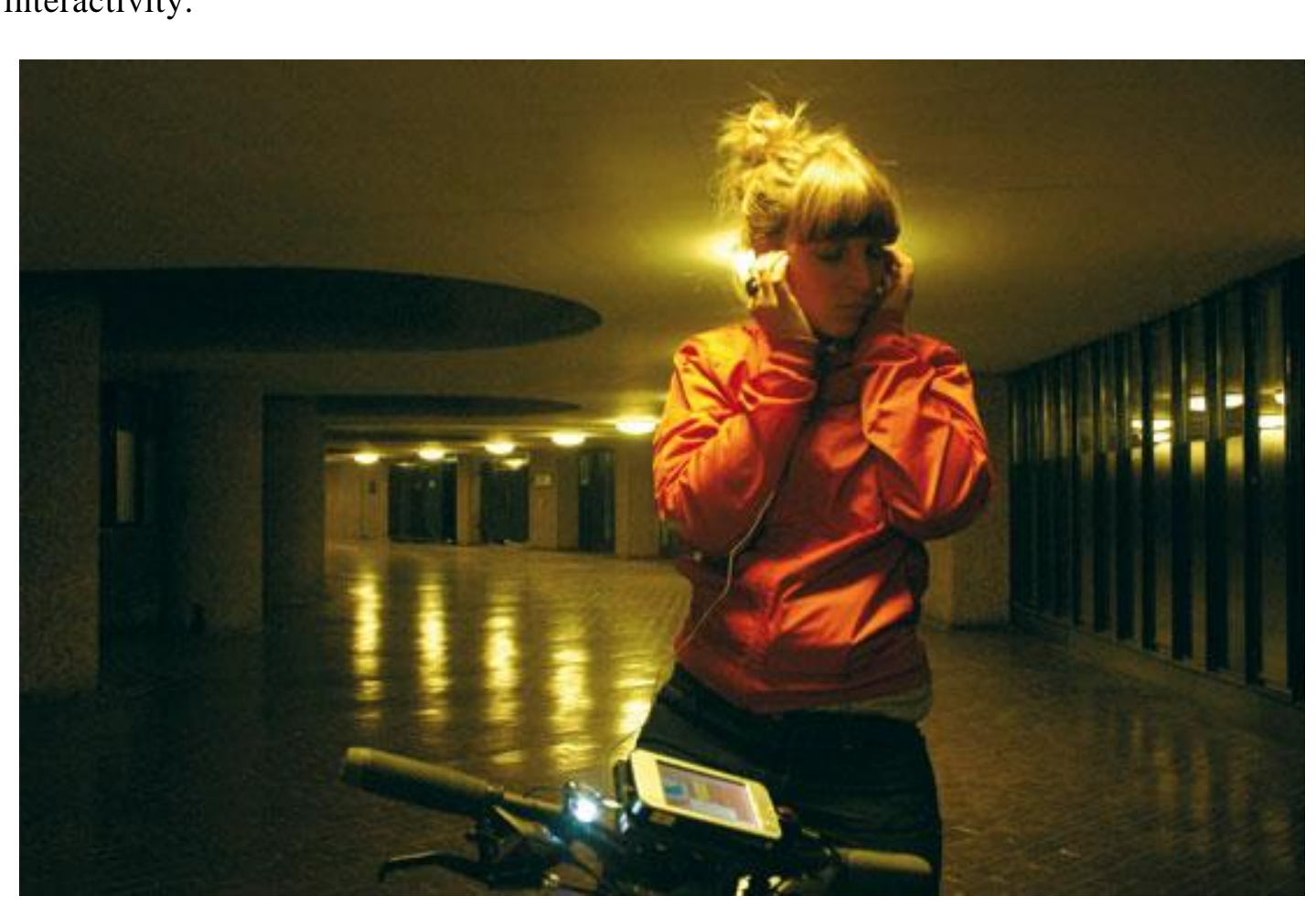

Figure 6. Rider Spoke's Participant using the Earplugs

\section{Conclusion}

Definitions of linear documentary have changed over time. The term still means different things to different people. We have followed Bill Nichol's approach [4], using a systemic definition that sees documentary as a set of relations forged between the 
author, the viewer, the media and what is around them. Those relations are changing with time; they are influenced by social, political and technological change. Those modes are ways to 'frame and organize (reality) into a text' and therefore they are symptomatic of a mode of 'negotiation' with reality. It is the idea of logics of negotiation of reality that I have retained to analyze digital interactive documentaries claiming that, once the user is demanded an active participation in the documentary, the negotiation happens through interactivity.

Until now, four main modes of interactivity have been used in interactive documentaries: hypertext, conversational, experiential and participatory. Those modes come from different visions of what the human-computer relation might be.

Interactivity is more than a simple action-reaction, human-machine process. Instead, it can be considered as a transformative force that creates infinite dynamic links between all the entities that are related to it and to each other. In this sense the interactive documentary becomes a relational object that has a life in itself.

\section{Acknowledgements}

This Paper is revised and expanded version of paper entitled Interactive Documentary as a New media presented at IUrC 2014, Budapest, Hungary, Aug.,14,2014.

\section{References}

[1] L. Manovichi, "The Language of New Media", The MIT Press, Boston, (2002).

[2] J. Aston and S. Gaudenzi, "Interactive Documentary Setting the field". Studies in Documentary film", vol. 6, no. 2, (2012).

[3] G. Davenport and M. Murtaugh, "ConText: Towands the Evolving Documentary, ACM Multimedia", (1995).

[4] N. Bill, "Introduction to Documentary", Indiana University Press, (2001).

[5] H. Ko, C. Romas, J. H. Abatwajy and S.Mohammeds, Editors. "Future Information and Computer Technology”, Proceedings of International Conferences, IUrC, SUComS, (2014) August 14-17, Budapest, Hungary.

[6] N. Kim, S. Kim and J.Y. Lee, International Journal of Multimedia and Ubiquitous Engineering, vol. 8 , no. $5,(\mathbf{2 0 1 3})$

[7] D. Galloway, K McAlpine, P. Harris, in Journal of Media Practice, vol. 8, no. 3, (2009), pp. 325-339.

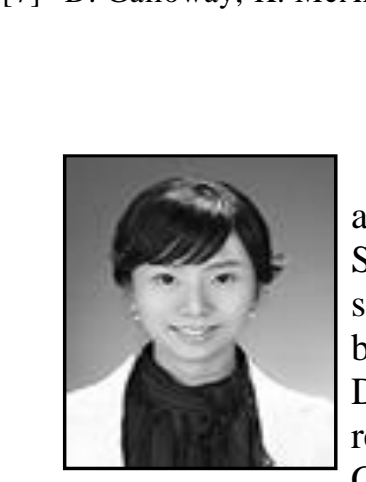

\section{Authors}

Nayun Kim, she received B.S. degrees in Russian and Journalism and Broadcasting in 2006 and M.S. degree in Cultural Contents Studies in 2011 from Hankuk University of Foreign Studies. Currently, she is pursuing a doctorate in the same course. Since 2011, she has been in charge of film industry support \& development and DMZ Documentary Film Festival at Gyeonggi Provincial Office. Her current research interests include Cultural Industry Policy, Next Generation Contents, Cultural Resource and Regional Cultural Contents. 


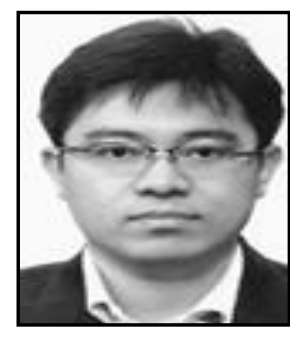

Sangheon Kim, he received B.S., M.S., and Ph.D. degrees in Computer Science from Soongsil University, Korea in 1992, 1994, and 2000 respectively. Since 2000, he has been a director of R\&D for NuriMedia, a Knowledge Contents Company in Seoul, Korea. Since 2006, he was an adjunct professor of Hankuk University of Foreign Studies, Department of Global Culture and Contents. His current research interests include Cultural Informatics, Cultural heritage and museum informatics.

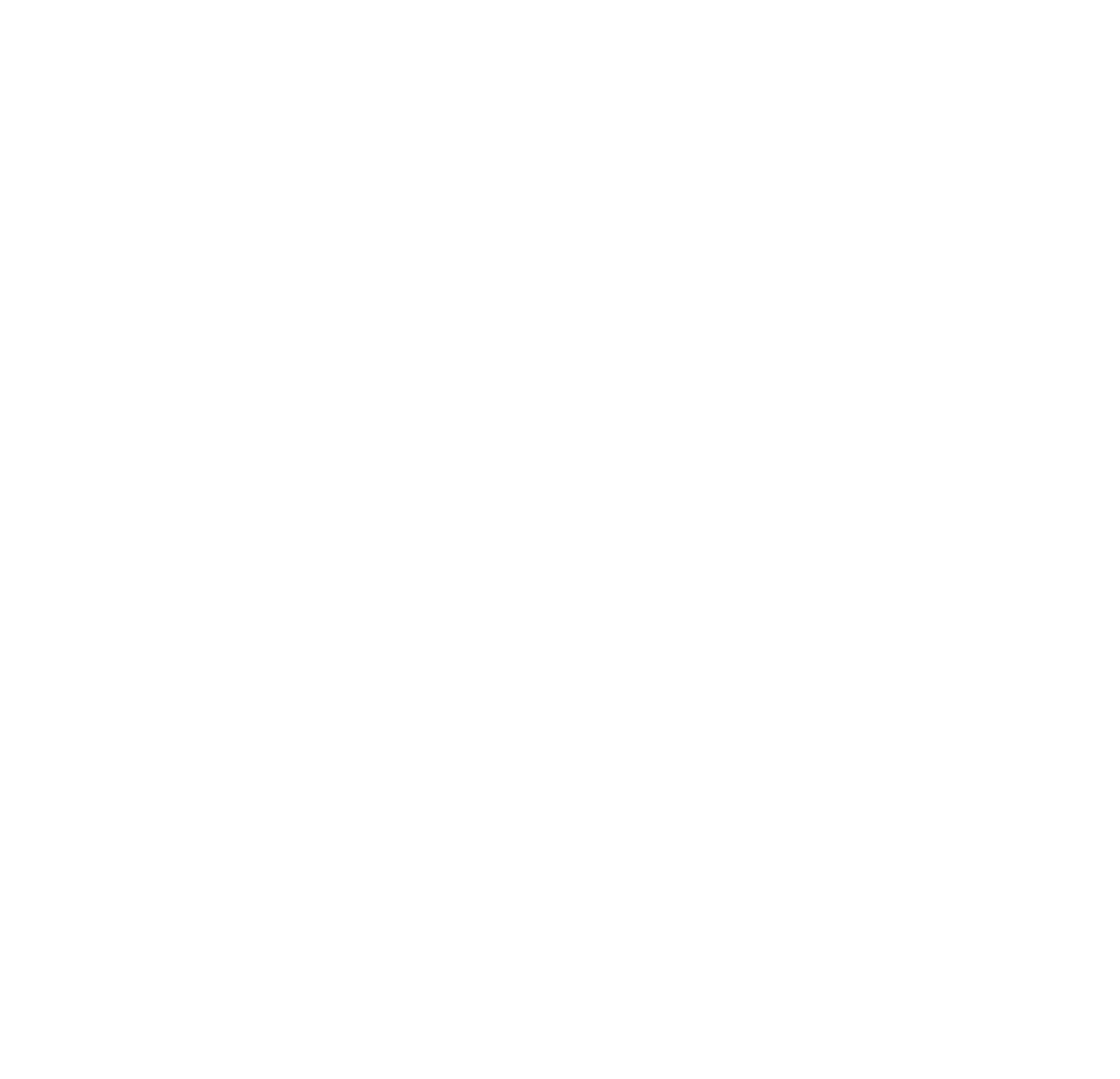

